\title{
Permanence of a Lotka-Volterra Predator-Prey Model with Feedback Controls and Prey Diffusion
}

\author{
Shuang Pan ${ }^{1,2}$, Yonghong $\mathrm{Li}^{2, *}$, Changyou Wang ${ }^{2, *}$ \\ ${ }^{1}$ College of Automation, Chongqing University of Posts and Telecommunications, Chongqing, P. R. China \\ ${ }^{2}$ Institute of Applied Mathematics, Chongqing University of Posts and Telecommunications, Chongqing, P. R. China
}

Email address:

liyh@cqupt.edu.cn (Yonghong Li), wangcy@cqupt.edu.cn (Changyou Wang)

${ }^{*}$ Corresponding author

To cite this article:

Shuang Pan, Yonghong Li, Changyou Wang. Permanence of a Lotka-Volterra Predator-Prey Model with Feedback Controls and Prey Diffusion. Mathematics Letters. Vol. 4, No. 1, 2018, pp. 6-13. doi: 10.11648/j.ml.20180401.12

Received: January 19, 2018; Accepted: February 6, 2018; Published: February 28, 2018

\begin{abstract}
This paper is concerned with a multi-delay three-species predator-prey model with feedback controls and prey diffusion. By developing some new analysis techniques and using the comparison principle of differential equations, we obtained some new sufficient conditions which ensure the system to be permanent.
\end{abstract}

Keywords: Predator-Prey Model, Feedback Control, Time Delay, Diffusion, Permanence

\section{Introduction}

From the biological aspects, it is important to obtain the conditions which ensure all species in multi-species community are permanent and global attractive. The Lotka-Volterra predator-prey model is one of the important interactions among species, which is commonly seen in the real world. In fact, diffusion often occurs in an ecological environment, that is to say, species can diffuse between patches. As a prey species which grows in weak environment, spread to an advantageous environment is conducive to maintaining the permanence and global attractive of the species. Therefore, the diffusion term is added to the model to describe accurately the interrelationships among species (see, [1-6]).

Song and Chen in [1] studied the following two-species predator-prey system with diffusion

$$
\left\{\begin{array}{l}
\dot{x}_{1}=x_{1}\left[a_{1}(t)-b_{1}(t) x_{1}-c(t) y\right]+D_{1}(t)\left(x_{2}-x_{1}\right), \\
\dot{x}_{2}=x_{2}\left[a_{2}(t)-b_{2}(t) x_{2}\right]+D_{2}(t)\left(x_{1}-x_{2}\right), \\
\dot{y}=y\left[-d(t)+e(t) x_{1}-q(t) y\right],
\end{array}\right.
$$

where $x_{1}$ and $y$ are population density of prey species $x$ and predator species $y$ in patch 1 , and $x_{2}$ is the density of prey species $x$ in patch 2 . Predator species $y$ is confined to patch 1 , while the prey species $x$ can diffuse between two patches. $D_{i}(t), i=1,2$ are diffusive coefficients of prey species $x$. It is proved that the system can be made persistent, further, if the system is a periodic system, it can have a strictly positive periodic orbit which is globally asymptotically stable under the appropriate conditions.

In 2010, Wei et al in [6] considered the following nonautonomous competitive Lotka-Volterra diffusion system

$$
\left\{\begin{array}{l}
\dot{x}_{1}=x_{1}\left[r_{1}(t)-a_{11}(t) x_{1}-a_{12}(t) y_{1}\right]+D_{1}(t)\left(x_{2}-x_{1}\right), \\
\dot{y}_{1}=y_{1}\left[r_{2}(t)-a_{21}(t) x_{1}-a_{22}(t) y_{1}\right]+D_{2}(t)\left(y_{2}-y_{1}\right), \\
\dot{x}_{2}=x_{2}\left[s_{1}(t)-b_{11}(t) x_{2}-b_{12}(t) y_{2}\right]+D_{1}(t)\left(x_{1}-x_{2}\right), \\
\dot{y}_{2}=y_{2}\left[s_{2}(t)-b_{21}(t) x_{2}-b_{22}(t) y_{2}\right]+D_{2}(t)\left(y_{1}-y_{2}\right),
\end{array}\right.
$$

They obtained a unique positive periodic solution which is globally asymptotically stable of the system under suitable conditions by constructing Liapunov function.

The phenomenon of time delay is common and inevitable in nature, a number of models in ecology can be formulated as systems of differential equations with time delays. Therefore, more realistic models of population interactions should take into the effect of time delays. In general, delay differential equations exhibit much more complicated dynamics than ordinary differential equations since a time delay could cause a stable equilibrium to become unstable 
and cause the population to fluctuate. In [7], the authors considered the following three-species Lotka-Volterra type competitive- mutualism systems with discrete time delay, and some sufficient conditions on the permanence of species and the global attractivity of the system are established.

$$
\left\{\begin{array}{l}
\dot{x}_{1}(t)=x_{1}(t)\left[r_{1}(t)-a_{11}^{1}(t) x_{1}(t-\tau)-a_{11}^{2}(t) x_{1}(t-2 \tau)-a_{12}(t) x_{2}(t-2 \tau)+a_{13}(t) x_{3}(t-\tau)\right] \\
\dot{x}_{2}(t)=x_{2}(t)\left[r_{2}(t)-a_{21}(t) x_{1}(t-2 \tau)-a_{22}^{1}(t) x_{2}(t-\tau)-a_{22}^{2}(t) x_{2}(t-2 \tau)+a_{23}(t) x_{3}(t-\tau)\right] \\
\dot{x}_{3}(t)=x_{3}(t)\left[r_{3}(t)+a_{31}(t) x_{1}(t-\tau)+a_{32}(t) x_{2}(t-\tau)-a_{33}^{1}(t) x_{3}(t)-a_{33}^{2}(t) x_{3}(t-\tau)\right]
\end{array}\right.
$$

Moreover, some researchers have been focused on the combined effects of dispersion and time- delays, in 2004, Xu et al [8] studied the following Lotka-Volterra predator-prey model with dispersion and time-delays.

$$
\left\{\begin{array}{l}
\dot{x}_{1}(t)=x_{1}(t)\left[r_{1}(t)-a_{11}(t) x_{1}(t)-a_{13}(t) y_{3}(t)\right]+D_{1}(t)\left(x_{2}(t)-x_{1}(t)\right) \\
\dot{x}_{2}(t)=x_{2}(t)\left[r_{2}(t)-a_{22}(t) x_{2}(t)\right]+D_{2}(t)\left(x_{1}(t)-x_{2}(t)\right) \\
\dot{y}_{3}(t)=y_{3}(t)\left[-r_{3}(t)+a_{31}(t) x_{1}\left(t-\tau_{1}\right)-a_{33}(t) y_{3}\left(t-\tau_{2}\right)\right]
\end{array}\right.
$$

where $x_{1}(t)$ and $y(t)$ denote the densities of species $x$ and $y$ in patch $1, x_{2}(t)$ denotes the density of species $x$ in patch 2. Predator species is confined to patch 1 while the prey species can disperse between two patches. $D_{i}(t), i=1,2$ are dispersion rate of prey species $x . \tau_{1}, \tau_{2}$ are positive constants.

In [9], Zhou, Shi and Song considered the following nonautonomous predator-prey model with nonlinear diffusion and time-delays. And they obtained some sufficient conditions for the permanence and global stability of the model. More work on diffusion and time-delays can be found in (cf, [10-12] and the references cited therein).

$$
\left\{\begin{array}{l}
\dot{x}_{1}(t)=x_{1}(t)\left[r_{1}(t)-k_{1}(t) x_{1}(t)-a_{1}(t) x_{3}(t)\right]+D_{1}(t) x_{1}(t)\left(x_{2}(t)-x_{1}(t)\right) \\
\dot{x}_{2}(t)=x_{2}(t)\left[r_{2}(t)-k_{2}(t) x_{2}(t)-a_{2}(t) x_{3}(t)\right]+D_{2}(t) x_{2}(t)\left(x_{1}(t)-x_{2}(t)\right) \\
\dot{x}_{3}(t)=-s(t) x_{3}(t)-b(t) x_{3}^{2}(t)+c_{1}(t) x_{3}(t-\tau) x_{1}(t-\tau)+c_{2}(t) x_{3}(t-\tau) x_{2}(t-\tau)
\end{array}\right.
$$

On the other hand, in some situation, people may wish to change the conditions of the system stability but to keep its stability still. This is of significance in the control of ecology balance. One of the methods for the realization of it is to alter the system structurally by introducing some feedback control variables so as to get a population stabilizing at another condition. The realization of the feedback control mechanism might be implemented by means of some biological control scheme or by harvesting procedure. In fact, during the last decade, the dynamical behavior of species for the various Lotka-Volterra systems with feedback controls have been studied in many articles, for example, see [13-16] and the references cited therein. Many important and interesting research results and methods are offered.

In 2003, K. Gopalsamy et al [13] studied the following two species competition system with feedback controls

$$
\left\{\begin{array}{l}
\frac{d x_{1}(t)}{d t}=x_{1}(t)\left[b_{1}-a_{11} x_{1}(t)-a_{12} x_{2}(t)-\alpha_{1} u_{1}(t)\right], \\
\frac{d x_{2}(t)}{d t}=x_{2}(t)\left[b_{2}-a_{21} x_{1}(t)-a_{22} x_{2}(t)-\alpha_{2} u_{2}(t)\right], \\
\frac{d u_{1}(t)}{d t}=-\eta_{1} u_{1}(t)+a_{1} x_{1}(t), \\
\frac{d u_{2}(t)}{d t}=-\eta_{2} u_{2}(t)+a_{2} x_{2}(t),
\end{array}\right.
$$

where $b_{i}, a_{i, j}, \alpha_{i}, \eta_{i}, a_{i}(i, j=1,2)$ are positive constants, $u_{i}(t), i=1,2$ are the indirect control variables. And they obtained some conditions for the existence of a globally attracting positive equilibrium of the system. In [16], Chen et al proposed the following Lotka-volterra predator-prey system with feedback controls

$$
\left\{\begin{array}{l}
\frac{d x_{1}(t)}{d t}=x_{1}(t)\left(a_{1}-b_{1} x_{1}(t)-c_{1} x_{2}(t)-\alpha_{1} u_{1}(t)\right), \\
\frac{d x_{2}(t)}{d t}=x_{2}(t)\left(-a_{2}+b_{2} x_{1}(t)-\alpha_{2} u_{2}(t)\right) \\
\frac{d u_{1}(t)}{d t}=-e_{1} u_{1}(t)+f_{1} x_{1}(t) \\
\frac{d u_{2}(t)}{d t}=-e_{2} u_{2}(t)+f_{2} x_{2}(t)
\end{array}\right.
$$

They by constructing two suitable Lyapunov functions, sufficient conditions which guarantee the global attractivity of the positive equilibrium and boundary equilibrium are obtained. They results show that enough large feedback controls could lead to the extinction of the predator species.

Moreover, in recent years, attention has been given to some ecosystem models with both feedback control and time delay or diffusions (see, [17-20]). In order to show that whether the feedback control variables play an essential role on the persistent property of Lotka-Volterra cooperative systems or not, $\mathrm{Xu}$ and Chen in [19] established and studied the following 
system with time delay and feedback control.

$$
\left\{\begin{array}{l}
\dot{x}_{1}(t)=x_{1}(t)\left[r_{1}(t)-a_{1}(t) x_{1}(t)-a_{11}(t) x_{1}(t-\tau)+a_{12}(t) x_{2}(t-\tau)-b_{1}(t) u_{1}\left(t-\sigma_{1}\right)\right], \\
\dot{x}_{2}(t)=x_{2}(t)\left[r_{2}(t)-a_{2}(t) x_{2}(t)+a_{21}(t) x_{1}(t)-a_{22}(t) x_{2}(t-\tau)-b_{2}(t) u_{2}\left(t-\sigma_{2}\right)\right], \\
\dot{u}_{1}(t)=-c_{1}(t) u_{1}(t)+d_{1}(t) x_{1}\left(t-\eta_{1}\right), \\
\dot{u}_{2}(t)=-c_{2}(t) u_{2}(t)+d_{2}(t) x_{2}\left(t-\eta_{2}\right),
\end{array}\right.
$$

where $x_{i}(t)$ denotes the density of $i$-species cooperative species, is the control variable, $i=1,2 . \quad r_{i}(t), a_{i}(t), b_{i}(t), c_{i}(t)$, $d_{i}(t), a_{i j}(t),(i=1,2)$ are all continuous, real-valued functions which are bounded above and below by positive constants. $\tau, \sigma_{i}, \eta_{i}, i=1,2$ are positive constants. They obtained some new sufficient conditions which ensure the system to be permanent, and show that feedback control variables have no influence on the permanence of the system.

To eliminate the influence of the patch diffusion and feedback control on existence of periodic solution, Xie and Weng in [20] considered the following predator-prey model with patch-diffusion and feedback control

$$
\left\{\begin{array}{l}
\dot{x}_{1}(t)=x_{1}(t)\left[b_{1}(t)-a_{1}(t) x_{1}(t)-\frac{k_{1}(t) y_{1}(t)}{c(t) y(t)+x_{1}(t)}\right]+D_{1}(t)\left(x_{2}(t)-x_{1}(t)\right), \\
\dot{x}_{2}(t)=x_{2}(t)\left[b_{2}(t)-a_{2}(t) x_{2}(t)-\beta_{1}(t) u_{1}(t)\right]+D_{2}(t)\left(x_{1}(t)-x_{2}(t)\right), \\
\dot{y}(t)=y(t)\left[-r(t)-a_{3}(t) y(t)+\frac{k_{2}(t) x_{1}\left(t-\tau_{1}\right)}{c(t) y\left(t-\tau_{1}\right)+x_{1}\left(t-\tau_{1}\right)}-\beta_{2}(t) u_{2}(t)\right], \\
\dot{u}_{1}(t)=-\eta_{1}(t) u_{1}(t)+\xi_{1}(t) x_{2}\left(t-\tau_{2}\right), \\
\dot{u}_{2}(t)=-\eta_{2}(t) u_{2}(t)+\xi_{2}(t) y\left(t-\tau_{3}\right),
\end{array}\right.
$$

where $x_{i}(t)(i=1,2)$ denotes the density of prey species at time $t, y(t)$ is the predator species density at time $t$,

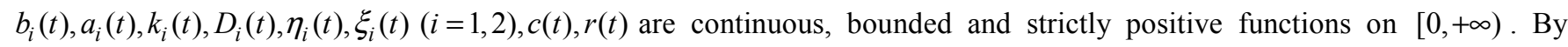
developing some new analysis methods, the existence of at least one positive periodic solution for this model is proved.

However, to the best of the authors' knowledge, to this day, still less scholars consider the general nonautonomous Lotka-Volterra predator-prey system with time-delays, prey diffusion and feedback controls. Based on system (4) and motivated by the above works, in this paper, we propose and investigate the following three-species Lotka-Volterra multi-delay predator-prey system with feedback controls and prey diffusions.

$$
\left\{\begin{array}{l}
\dot{x}_{1}(t)=x_{1}(t)\left[r_{1}(t)-a_{11}(t) x_{1}(t)-a_{13}(t) x_{3}(t)-d_{1}(t) u_{1}(t)\right]+D_{1}(t)\left[x_{2}(t)-x_{1}(t)\right], \\
\dot{x}_{2}(t)=x_{2}(t)\left[r_{2}(t)-a_{22}(t) x_{2}(t)-a_{23}(t) x_{3}(t)-d_{2}(t) u_{2}(t)\right]+D_{2}(t)\left[x_{1}(t)-x_{2}(t)\right], \\
\dot{x}_{3}(t)=x_{3}(t)\left[-r_{3}(t)+a_{31}(t) x_{1}\left(t-\tau_{1}\right)+a_{32}(t) x_{2}\left(t-\tau_{2}\right)-a_{33}(t) x_{3}\left(t-\tau_{3}\right)+d_{3}(t) u_{3}(t)\right], \\
\dot{u}_{1}(t)=e_{1}(t)-f_{1}(t) u_{1}(t)+q_{1}(t) x_{1}(t), \\
\dot{u}_{2}(t)=e_{2}(t)-f_{2}(t) u_{2}(t)+q_{2}(t) x_{2}(t), \\
\dot{u}_{3}(t)=e_{3}(t)-f_{3}(t) u_{3}(t)-q_{3}(t) x_{3}(t),
\end{array}\right.
$$

Due to biological interpretation of system (10), it is reasonable to consider only positive solution of (10), in other words, to take admissible initial conditions

$$
\begin{aligned}
& x_{i}(t)=\phi_{i}(t), i=1,2,3 \text { for } t \in[-\tau, 0) \text { and } \phi_{i}(0)>0, \\
& u_{i}(t)=\phi_{i}(t), i=1,2,3 \text { for } t>0 \text { and } \varphi_{i}(0)>0 .
\end{aligned}
$$

Obviously, the solutions of system (10) with the initial values (11) are positive for all $t \geq 0$. Where $x_{1}(t)$ and $x_{2}(t)$ denote the density of prey species in patch 1 and patch 2 , respectively, $x_{3}(t)$ is the density of predator species, while the prey can diffuse between two patches; $r_{i}(t)(i=1,2)$ denote the intrinsic growth rate of the prey species at patch 1 and patch $2, r_{3}(t)$ is the death rate of the predator; $a_{i i}(t)(i=1,2,3)$ denote the restriction density of the three species, respectively. $a_{13}(t)$ and $a_{23}(t)$ are the capturing rate of the predator, $a_{31}(t)$ and $a_{32}(t)$ are the conversion rate of nutrients of the predator; $D_{i}(t)(i=1,2)$ are the dispersion rate of prey species, $u_{i}(t)(i=1,2,3)$ are the feedback control terms; furthermore $a_{13}(t), a_{23}(t)$, 
$a_{31}(t), a_{32}(t), D_{1}(t), D_{2}(t), r_{i}(t), a_{i i}(t), d_{i}(t), e_{i}(t)$, and $f_{i}(t), q_{i}(t)$, $(i=1,2,3)$ are continuous, bounded and strictly positive functions on $[0,+\infty), \tau_{1}, \tau_{2}, \tau_{3}$ are positive constants. By developing a new analysis technique, the sufficient conditions are established for the permanence of the predator-prey model in this paper.

\section{Permanence}

For a continuous and bounded function $g(t)$ defined on $\left[t_{0},+\infty\right)$, we set

$$
g^{m}=\sup \left\{g(t) \mid t_{0}<t<+\infty\right\}, \quad g^{l}=\inf \left\{g(t) \mid t_{0}<t<+\infty\right\} .
$$

Definition 2.1. System (10) is called permanent, if there exist positive constants $M_{i}, m_{i}(i=1,2), \quad N_{i}, n_{i}(i=1,2,3)$ and $T$, such that

$$
\begin{aligned}
& m_{1} \leq x_{i}(t) \leq M_{1}, m_{2} \leq x_{3}(t) \leq M_{2}, i=1,2, \\
& n_{i} \leq u_{i}(t) \leq N_{i}, i=1,2,3,
\end{aligned}
$$

for any positive solution $Z(t)=\left(x_{1}(t), x_{2}(t), x_{3}(t), u_{1}(t), u_{2}(t)\right.$ ,$\left.u_{3}(t)\right)$ of system (10) as $t>T$.

As a direct corollary of Lemma 2.1 of Chen [21], we have

Lemma 2.1. If $a>0, b>0$ and $\dot{x} \geq b-a x$, when $t \geq 0$ and $x(0)>0$, we have

$$
\liminf _{t \rightarrow+\infty} x(t) \geq b / a
$$

If $a>0, b>0$ and $\dot{x} \leq b-a x$, when $t \geq 0$ and $x(0)>0$, we have

$$
\limsup _{t \rightarrow+\infty} x(t) \leq b / a
$$

As a direct corollary of Lemma 2.2 of Chen [21], we have

Lemma 2.2. If $a>0, b>0$ and $\dot{x} \geq x(b-a x)$, when $t \geq 0$ and $x(0)>0$, we have

$$
\liminf _{t \rightarrow+\infty} x(t) \geq b / a
$$

If $a>0, b>0$ and $\dot{x} \leq x(b-a x)$, when $t \geq 0$ and $x(0)>0$, we have

$$
\limsup _{t \rightarrow+\infty} x(t) \leq b / a
$$

Lemma 2.3 (see [22], Lemma 2.2). Assume that for $y(t)>0$, it holds that

$$
\dot{y}(t) \leq y(t)\left(\lambda-\sum_{l=0}^{m} \mu^{l} y(t-l \tau)+D\right.
$$

with initial conditions $y(t)=\phi(t) \geq 0$ for $t \in[-m \tau, 0)$ and $\phi(0)>0$, where

$$
\lambda>0, \mu^{l} \geq 0, l=0,1,2, \cdots, m, \mu=\sum_{l=0}^{m} \mu^{l}>0 \text { and } D \geq 0,
$$

are constants, Then there exist a positive constant $M_{y}<+\infty$ such that

$$
\limsup _{t \rightarrow+\infty} y(t) \leq M_{y}=-\frac{D}{\lambda}+\left(\frac{D}{\lambda}+y^{*}\right) \exp (\lambda m \tau)<+\infty,
$$

where $y=y^{*}$ is the unique solution of $y(\lambda-\mu y)+D=0$.

Lemma 2.4 (see [22], Lemma 2.3). Assume that for $y(t)>0$, it holds that

$$
\dot{y}(t) \geq y(t)\left[\lambda-\sum_{l=0}^{m} \mu^{l} y(t-l \tau)\right]
$$

If (12) holds, then, there exists a positive constant $m_{y}>0$ such that for $\mu=\sum_{l=0}^{m} \mu^{l}>0$,

$$
\liminf _{t \rightarrow+\infty} y(t) \geq m_{y}=\frac{\lambda}{\mu} \exp \left\{\left(\lambda-\mu M_{y}\right) m \tau\right\}>0 .
$$

For system (10), we let

$$
\begin{aligned}
& M_{1}=\max \left\{\frac{r_{1}^{m}}{a_{11}^{l}}, \frac{r_{2}^{m}}{a_{22}^{l}}\right\}, M_{2}=x_{3}^{*} \exp \left\{\left[\left(a_{31}^{m}+a_{32}^{m}\right) M_{1}+d_{3}^{m} N_{3}\right] \tau_{3}\right\}, \\
& N_{1}=\frac{e_{1}^{m}+q_{1}^{m} M_{1}}{f_{1}^{l}}, N_{2}=\frac{e_{2}^{m}+q_{2}^{m} M_{1}}{f_{2}^{l}}, N_{3}=\frac{e_{3}^{m}}{f_{3}^{l}}, \\
& m_{1}=\min \left\{\frac{r_{1}^{l}-a_{13}^{m} M_{2}-d_{1}^{m} N_{1}}{a_{11}^{m}}, \frac{r_{2}^{l}-a_{23}^{m} M_{2}-d_{2}^{m} N_{2}}{a_{22}^{m}}\right\}, \\
& m_{2}=\frac{\left(a_{31}^{l}+a_{32}^{l}\right) m_{1}-r_{3}^{m}}{a_{33}^{m}} \exp \left\{\left(\left(a_{31}^{l}+a_{32}^{l}\right) m_{1}-r_{3}^{m}-a_{33}^{m} M_{2}\right) \tau_{3}\right\}, \\
& n_{1}=\frac{e_{1}^{l}+q_{1}^{l} m_{1}}{f_{1}^{m}}, n_{2}=\frac{e_{2}^{l}+q_{2}^{l} m_{1}}{f_{2}^{m}}, n_{3}=\frac{e_{3}^{l}-q_{3}^{m} M_{2}}{f_{3}^{m}} .
\end{aligned}
$$

and $x_{3}^{*}$ is the unique positive solution of the following equation

$$
x_{3}(t)\left(\left(a_{31}^{m}+a_{32}^{m}\right) M_{1}+d_{3}^{m} N_{3}-a_{33}^{l} x_{3}(t)\right)=0 .
$$

Theorem 2.1. Assume that the system (10) satisfies the following conditions

$$
\begin{aligned}
& \left(H_{1}\right) r_{1}^{l}>a_{13}^{m} M_{2}+d_{1}^{m} N_{1},\left(H_{2}\right) r_{2}^{l}>a_{23}^{m} M_{2}+d_{2}^{m} N_{2}, \\
& \left(H_{3}\right) r_{3}^{m}<\left(a_{31}^{l}+a_{32}^{l}\right) m_{1},\left(H_{4}\right) e_{3}^{l}>q_{3}^{m} M_{2} .
\end{aligned}
$$

then the system (10) is permanent.

Proof. According to the first and the second equations of system (10), we define $W_{1}(t)=\max \left\{x_{1}(t), x_{2}(t)\right\}$ and calculate the upper right derivative of $W_{1}(t)$ along the positive solution of system (10), we have that

(P1) If $x_{1}(t) \geq x_{2}(t)$, then 


$$
\begin{aligned}
D^{+} W_{1}(t) & =\dot{x}_{1}(t)=x_{1}(t)\left[r_{1}(t)-a_{11}(t) x_{1}(t)-a_{13}(t) x_{3}(t)-d_{1}(t) u_{1}(t)\right]+D_{1}(t)\left[x_{2}(t)-x_{1}(t)\right] \\
& \leq x_{1}(t)\left[r_{1}(t)-a_{11}(t) x_{1}(t)\right] \leq x_{1}(t)\left[r_{1}^{m}-a_{11}^{l} x_{1}(t)\right]=W_{1}(t)\left[r_{1}^{m}-a_{11}^{l} W_{1}(t)\right] .
\end{aligned}
$$

(P2) If $x_{1}(t) \leq x_{2}(t)$, then

$$
\begin{aligned}
D^{+} W_{1}(t) & =\dot{x}_{2}(t)=x_{2}(t)\left[r_{2}(t)-a_{22}(t) x_{2}(t)-a_{23}(t) x_{3}(t)-d_{2}(t) u_{2}(t)\right]+D_{2}(t)\left[x_{1}(t)-x_{2}(t)\right] \\
& \leq x_{2}(t)\left[r_{2}(t)-a_{22}(t) x_{2}(t)\right] \leq x_{2}(t)\left[r_{2}^{m}-a_{22}^{l} x_{2}(t)\right]=W_{1}(t)\left[r_{2}^{m}-a_{22}^{l} W_{1}(t)\right] .
\end{aligned}
$$

From (P1) and (P2), we have

$$
D^{+} W_{1}(t) \leq W_{1}(t)\left[r_{i}^{m}-a_{i i}^{l} W_{1}(t)\right], i=1,2 .
$$

By (13) we can derive

(A) If $W_{1}(0)=\max \left\{x_{1}(0), x_{2}(0)\right\} \leq M_{1}$, then $\max \left\{x_{1}(t)\right.$,

$\left.x_{2}(t)\right\} \leq M_{1}, t \geq 0$.

(B) If $W_{1}(0)=\max \left\{x_{1}(0), x_{2}(0)\right\}>M_{1}$, take appropriate $\alpha>0$, we have the following three possibilities:

(a) $W_{1}(0)=x_{1}(0)>M_{1},\left(x_{1}(0)>x_{2}(0)\right)$;

(b) $W_{1}(0)=x_{2}(0)>M_{1},\left(x_{1}(0)<x_{2}(0)\right)$;

(c) $W_{1}(0)=x_{1}(0)=x_{2}(0)>M_{1}$.

If (a) holds, then there exists $\varepsilon>0, t \in[0, \varepsilon)$, we have $W_{1}(t)=x_{1}(t)>M_{1}$, then we get that

$$
D^{+} W_{1}(t)=\dot{x}_{1}(t) \leq a_{11}^{l} W_{1}(t)\left[\frac{r_{1}^{m}}{a_{11}^{l}}-W_{1}(t)\right] \leq-\alpha<0 .
$$

If (b) holds, then there exists $\varepsilon>0, t \in[0, \varepsilon)$, and $W_{1}(t)=x_{2}(t)>M_{1}$, then

$$
\begin{aligned}
D^{+} W_{1}(t)=\dot{x}_{2}(t) \leq a_{22}^{l} W_{1}(t)\left[\frac{r_{2}^{m}}{a_{22}^{l}}-W_{1}(t)\right] \leq-\alpha<0 . \\
\dot{u}_{3}(t)=e_{3}(t)-f_{3}(t) u_{3}(t)-q_{3}(t) x_{3}(t) \leq e_{3}(t)-f_{3}(t) u_{3}(t) \leq e_{3}^{m}-f_{3}^{l} u_{3}(t) .
\end{aligned}
$$

And by Lemma 2.1, we have

$$
\limsup _{t \rightarrow+\infty} u_{3}(t) \leq N_{3}=\frac{e_{3}^{m}}{f_{3}^{l}}
$$

By the third equation of system (10), we have

$$
\begin{aligned}
\dot{x}_{3}(t) & =x_{3}(t)\left[-r_{3}(t)+a_{31}(t) x_{1}\left(t-\tau_{1}\right)+a_{32}(t) x_{2}\left(t-\tau_{2}\right)-a_{33}(t) x_{3}\left(t-\tau_{3}\right)+d_{3}(t) u_{3}(t)\right] \\
& \leq x_{3}(t)\left[a_{31}(t) x_{1}\left(t-\tau_{1}\right)+a_{32}(t) x_{2}\left(t-\tau_{2}\right)-a_{33}(t) x_{3}\left(t-\tau_{3}\right)+d_{3}(t) u_{3}(t)\right] \\
& \leq x_{3}(t)\left[\left(a_{31}^{m}+a_{32}^{m}\right) M_{1}+d_{3}^{m} N_{3}-a_{33}(t) x_{3}\left(t-\tau_{3}\right)\right] .
\end{aligned}
$$

And from the Lemma 2.3, we derive that

$\limsup x_{3}(t) \leq M_{2}=x_{3}^{*} \exp \left\{\left[\left(a_{31}^{m}+a_{32}^{m}\right) M_{1}+d_{3}^{m} N_{3}\right] \tau_{3}\right\}$.

where $x_{3}^{*}$ is the unique positive solution of the following equation

$$
x_{3}(t)\left(\left(a_{31}^{m}+a_{32}^{m}\right) M_{1}+d_{3}^{m} N_{3}-a_{33}^{l} x_{3}(t)\right)=0 .
$$

For the fourth and the fifth of system (10), we have

$$
\begin{aligned}
& \dot{u}_{1}(t)=e_{1}(t)-f_{1}(t) u_{1}(t)+q_{1}(t) x_{1}(t) \leq e_{1}^{m}-f_{1}^{l} u_{1}(t)+q_{1}^{m} M_{1}, \\
& \dot{u}_{2}(t)=e_{2}(t)-f_{2}(t) u_{2}(t)+q_{2}(t) x_{2}(t) \leq e_{2}^{m}-f_{2}^{l} u_{2}(t)+q_{2}^{m} M_{1} .
\end{aligned}
$$

By Lemma 2.1, we can get 


$$
\begin{aligned}
& \limsup _{t \rightarrow+\infty} u_{1}(t) \leq N_{1}=\frac{e_{1}^{m}+q_{1}^{m} M_{1}}{f_{1}^{l}} . \\
& \limsup _{t \rightarrow+\infty} u_{2}(t) \leq N_{2}=\frac{e_{2}^{m}+q_{2}^{m} M_{1}}{f_{2}^{l}} \\
& D_{+} W_{2}(t)=\dot{x}_{1}(t)=x_{1}(t)\left[r_{1}(t)-a_{11}(t) x_{1}(t)-a_{13}(t) x_{3}(t)-d_{1}(t) u_{1}(t)\right]+D_{1}(t)\left[x_{2}(t)-x_{1}(t)\right] \\
& \geq x_{1}(t)\left[r_{1}^{l}-a_{11}^{m} x_{1}(t)-a_{13}^{m} M_{2}-d_{1}^{m} N_{1}\right]=W_{2}(t)\left[r_{1}^{l}-a_{13}^{m} M_{2}-d_{1}^{m} N_{1}-a_{11}^{m} W_{2}(t)\right] .
\end{aligned}
$$

On the other hand, According to the first and the second equations of system (10), we define $W_{2}(t)=\min \left\{x_{1}(t), x_{2}(t)\right\}$, then calculating the lower right derivative of $W_{2}(t)$ along the

(2) If $x_{1}(t) \geq x_{2}(t)$, then

$$
\begin{aligned}
D_{+} W_{2}(t) & =\dot{x}_{2}(t)=x_{2}(t)\left[r_{2}(t)-a_{22}(t) x_{2}(t)-a_{23}(t) x_{3}(t)-d_{2}(t) u_{2}(t)\right]+D_{2}(t)\left[x_{1}(t)-x_{2}(t)\right] \\
& \geq x_{2}(t)\left[r_{2}^{l}-a_{22}^{m} x_{2}(t)-a_{23}^{m} M_{2}-d_{2}^{m} N_{2}\right]=W_{2}(t)\left[r_{2}^{l}-a_{23}^{m} M_{2}-d_{2}^{m} N_{2}-a_{22}^{m} W_{2}(t)\right] .
\end{aligned}
$$

From (1) and (2), it is easy to obtain

$$
D_{+} W_{2}(t) \geq W_{i}(t)\left[r_{i}^{l}-a_{i 3}^{m} M_{2}-d_{i}^{m} N_{i}-a_{i i}^{m} W_{i}(t)\right], i=1,2 .
$$

By (20), we can obtain the following

(i) if $W_{2}(0)=\min \left\{x_{1}(0), x_{2}(0)\right\} \geq m_{1}$, then $\min \left\{x_{1}(t), x_{2}(t)\right\} \geq m_{1}, t \geq 0$.

(ii) if $W_{2}(0)=\min \left\{x_{1}(0), x_{2}(0)\right\}<m_{1}$, take appropriate $\alpha>0$, we have

(a) $W_{2}(0)=x_{1}(0)<m_{1},\left(x_{1}(0)<x_{2}(0)\right)$;

(b) $W_{2}(0)=x_{2}(0)<m_{1},\left(x_{1}(0)>x_{2}(0)\right)$;

(c) $W_{2}(0)=x_{1}(0)=x_{2}(0)<m_{1}$.

If (a) holds, there exists $\varepsilon>0$ such that if $t \in[0, \varepsilon)$, we have $W_{2}(t)=x_{1}(t)<m_{1}$, then

$$
D_{+} W_{2}(t)=\dot{x}_{1}(t) \geq a_{11}^{m} W_{2}(t)\left[\frac{r_{1}^{l}-a_{13}^{m} M_{2}-d_{1}^{m} N_{1}}{a_{11}^{m}}-W_{2}(t)\right] \geq \alpha>0 .
$$

If (b) holds, there exists $\varepsilon>0$ if $t \in[0, \varepsilon)$, we have $W_{2}(t)=x_{2}(t)<m_{1}$, then

$$
D_{+} W_{2}(t)=\dot{x}_{2}(t) \geq a_{22}^{m} W_{2}(t)\left[\frac{r_{2}^{l}-a_{23}^{m} M_{2}-d_{2}^{m} N_{2}}{a_{22}^{m}}-W_{2}(t)\right] \geq \alpha>0 .
$$

If (c) holds, in the same way, we have

$$
D_{+} W_{2}(t)=\dot{x}_{i}(t) \geq a_{i i}^{m} W_{2}(t)\left[\frac{r_{i}^{l}-a_{i 3}^{m} M_{2}-d_{i}^{m} N_{i}}{a_{i i}^{m}}-W_{2}(t)\right] \geq \alpha>0, i=1 \text { or } 2 .
$$

From (a), (b), (c), we can get that if $W_{2}(0)<m_{1}$, then $W_{2}(t)$ is strictly monotonously increasing with speed $\alpha$. Then there exists $T_{2}>0$ such that if $t \geq T_{2}$, we have $W_{2}(t)=\min \left\{x_{1}(t), x_{2}(t)\right\} \geq m_{1}$.

That is

$$
\begin{aligned}
& \liminf _{t \rightarrow+\infty} x_{1}(t) \geq m_{1}=\min \left\{\frac{r_{1}^{l}-a_{13}^{m} M_{2}-d_{1}^{m} N_{1}}{a_{11}^{m}}, \frac{r_{2}^{l}-a_{23}^{m} M_{2}-d_{2}^{m} N_{2}}{a_{22}^{m}}\right\} . \\
& \liminf _{t \rightarrow+\infty} x_{2}(t) \geq m_{1}=\min \left\{\frac{r_{1}^{l}-a_{13}^{m} M_{2}-d_{1}^{m} N_{1}}{a_{11}^{m}}, \frac{r_{2}^{l}-a_{23}^{m} M_{2}-d_{2}^{m} N_{2}}{a_{22}^{m}}\right\} .
\end{aligned}
$$

For the third equation of system (10), we have 


$$
\begin{aligned}
\dot{x}_{3}(t) & =x_{3}(t)\left[-r_{3}(t)+a_{31}(t) x_{1}\left(t-\tau_{1}\right)+a_{32}(t) x_{2}\left(t-\tau_{2}\right)-a_{33}(t) x_{3}\left(t-\tau_{3}\right)+d_{3}(t) u_{3}(t)\right] \\
& \geq x_{3}(t)\left[-r_{3}(t)+a_{31}(t) x_{1}\left(t-\tau_{1}\right)+a_{32}(t) x_{2}\left(t-\tau_{2}\right)-a_{33}(t) x_{3}\left(t-\tau_{3}\right)\right] \\
& \geq x_{3}(t)\left[-r_{3}^{m}+a_{31}^{l} m_{1}+a_{32}^{l} m_{1}-a_{33}^{m} x_{3}\left(t-\tau_{3}\right)\right] .
\end{aligned}
$$

By Lemma 2.4, we have

$$
\liminf _{t \rightarrow+\infty} x_{3}(t) \geq m_{2}=\frac{\left(a_{31}^{l}+a_{32}^{l}\right) m_{1}-r_{3}^{m}}{a_{33}^{m}} \exp \left\{\left(\left(a_{31}^{l}+a_{32}^{l}\right) m_{1}-r_{3}^{m}-a_{33}^{m} M_{2}\right) \tau_{3}\right\} .
$$

From the fourth and the fifth of system (10), we have

$$
\begin{aligned}
& \dot{u}_{1}(t)=e_{1}(t)-f_{1}(t) u_{1}(t)+q_{1}(t) x_{1}(t) \geq e_{1}^{l}-f_{1}^{m} u_{1}(t)+q_{1}^{l} m_{1}, \\
& \dot{u}_{2}(t)=e_{2}(t)-f_{2}(t) u_{2}(t)+q_{2}(t) x_{2}(t) \geq e_{2}^{l}-f_{2}^{m} u_{2}(t)+q_{2}^{l} m_{1} .
\end{aligned}
$$

And by Lemma 2.1, we can get

$$
\begin{aligned}
& \liminf _{t \rightarrow+\infty} u_{1}(t) \geq n_{1}=\frac{e_{1}^{l}+q_{1}^{l} m_{1}}{f_{1}^{m}} . \\
& \liminf _{t \rightarrow+\infty} u_{2}(t) \geq n_{2}=\frac{e_{2}^{l}+q_{2}^{l} m_{1}}{f_{2}^{m}} .
\end{aligned}
$$

According to the sixth equation of system (10), we have

$\dot{u}_{3}(t)=e_{3}(t)-f_{3}(t) u_{3}(t)-q_{3}(t) x_{3}(t) \geq e_{3}^{l}-f_{3}^{m} u_{3}(t)-q_{3}^{m} M_{2}$.

From Lemma 2.1,

$$
\liminf _{t \rightarrow+\infty} u_{3}(t) \geq n_{3}=\frac{e_{3}^{l}-q_{3}^{m} M_{2}}{f_{3}^{m}} .
$$

From (14)-(19) and (21)-(26), this ends the proof of Theorem 2.1.

\section{Conclusion}

This paper presents the use of some new analysis techniques and the comparison principle of differential equations. This method is a powerful tool for solving nonlinear differential equations in mathematical physics, chemistry and engineering etc. We have dealt with the problem of positive solution for a multi-delay three-species predator-prey model with feedback controls and prey diffusion. The general sufficient conditions have been obtained to ensure the permanence of positive solution for the predator-prey model. In particular, the sufficient conditions that we obtained are very simple, which provide flexibility for the application and analysis of the Lotka-Volterra predator-prey system.

\section{Acknowledgements}

This work is supported Science Fund for Distinguished Young Scholars (cstc2014jcyjjq40004) of China, the National Nature Science Fund (Project nos.11372366 and 61503053) of China, the Natural Science Foundation Project of CQ CSTC
(Grant nos. cstc2015jcyjA00034, cstc2015jcyjBX0135 and cstc2015jjA20016) of China.

\section{References}

[1] SONG X, CHEN L. Persistence and periodic orbits for two-species predator-prey system with diffusion. Canadian Applied Mathematics Quarterly, 1998, 6 (3): 233-244.

[2] SONG X, CHEN L. Persistence and global stability for nonautonomous predator-prey system with diffusion and time delay. Computers \& Mathematics with Applications, 1998, 35 (6): 33-40.

[3] CUI J. The Effect of Dispersal on Permanence in a Predator-Prey Population Growth Model. Computers and Mathematics with Applications, 2002, 44 (8):1085-1097.

[4] CHEN F, XIE X. Permanence and Extinction in Nonlinear Single and Multiple Species System with Diffusion. Applied Mathematics and Computation, 2006, 177 (1): 410-426.

[5] ZHANG F, ZHAO X. Global Dynamics of a Nonautonomous Predator-Prey System with Dispersion. Mathematical Analysis, 2007, 14 (1): 81-87.

[6] WEI F, LIN Y, QUE L, et al. Periodic Solution and Global Stability for a Nonautonomous Competitive Lotka-Volterra Diffusion System. Applied Mathematics and Computation, 2010, 216 (10): 3097-3104.

[7] MUHAMMADHAJI A, TENG Z, REHIM M. Dynamical Behavior for a Class of Delayed Competitive-Mutulism Systems. Differential Equations and Dynamical Systems, 2015, 23 (3): 281-301.

[8] XU R, CHAPLAIN M, DAVIDSON F A. Periodic Solution of a Lotka-Volterra Predator-Prey Model with Dispersion and Time Delays. Applied Mathematics and Computation, 2004, 148 (2): 537-560.

[9] ZHOU X, SHI X, SONG X. Analysis of Nonautonomous Predator-Prey Model with Nonlinear Diffusion and Time Delay. Applied Mathematics and Computation, 2008, 196(1): 129-136.

[10] ZHANG Z, WANG Z. Periodic Solutions of a Two-Species Ratio-Dependent Predator-Prey System With TimeDelay in a Two-Patch Environment. Anziam Journal, 2003, 45 (2): 233-244.

[11] LIANG R, SHEN J. Positive Periodic Solutions for Impulsive Predator-Prey Model with Dispersion and Time Delays. Applied Mathematics and Computation, 2010, 217 (2): 661-676.

[12] MUHAMMADHAJI A, MAHEMUTI R, TENG Z. On a Periodic Predator-Prey System with Nonlinear Diffusion and Delays. Afrika Matematika, 2016, 27 (7-8): 1179-1197. 
[13] GOPALSAMY K, WENG P. Global Attractivity in a Competition System with Feedback Controls. Computers and Mathematics with Applications, 2003, 45 (4-5): 665-676.

[14] CHEN F. The Permanence and Global Attractivity of LotkaVolterra Competition System with Feedback Controls. Nonlinear Analysis: Real World Applications, 2006, 7 (1): 133-143.

[15] NIE L, TENGA Z, HU L, et al. Permanence and Stability in Nonautonomous Predator-Prey Lotka-Volterra Systems with Feedback Controls. Computers and Mathematics with Applications, 2009, 58 (3): 436-448.

[16] CHEN F, GONG X, PU L, et al. Dynamic Behaviors of a Lotka-Volterra Predator-Prey System with Feedback Controls. Journal of Biomathematics, 2015, 30 (2): 328-332. (In chinese).

[17] DING X, FANGFANGWANG. Positive Periodic Solution for a Semi-Ratio-Dependent Predator-Prey System with Diffusion and Time Delays. Nonlinear Analysis: RealWorld Applications, 2008, 9 (2): 239-249.
[18] GOPALSAMY K, WENG P. Feedback Regulation of Logistic Growth. International Journal of Mathematics \& Mathematical Sciences, 1993, 16 (1): 177-192.

[19] XU J, CHEN F. Permanence of a Lotka-Volterra Cooperative System with Time Delays and Feedback Controls. Communications in Mathematical Biology \& Neuroscience, 2015, 18. (2): 226-237.

[20] XIE W, WENG P. Existence of Periodic Solution for a Predator - Prey Model with Patch - Diffusion and Feedback Control. Journal of South China Normal University (Natural Science Edition), 2012, 44 (1): 42-47. (In Chinese).

[21] CHEN F. On a Nonlinear Nonautonomous Predator-Prey Model with Diffusion and Distributed Delay. Journal of Computational and Applied Mathematics, 2005, 180 (1): 33-49.

[22] NAKATA Y, MUROYA Y. Permanence for Nonautonomous Lotka-Volterra Cooperative Systems with Delays. Nonlinear Analysis: Real World Applications, 2010, 11(1): 528-534. 